\title{
Metodología teórica para analizar la congruencia entre representaciones de objetos matemáticos: el caso de los intervalos no acotados de la recta real
}

\author{
Theoretical methodology to analyze the degree of congruence \\ between representations of mathematical objects: the case of \\ the unbounded intervals of the real line
}

\author{
Cristina Pecharromán ${ }^{1}$ \\ Matías Arce ${ }^{2}$ \\ Laura Conejo ${ }^{3}$ \\ Tomás Ortega ${ }^{4}$
}

\begin{abstract}
Resumen: Siguiendo a Duval $(1999,2006)$, consideramos que la aprehensión de un concepto pasa por el uso comprensivo y espontáneo de sus representaciones, y que la habilidad para realizar conversiones entre registros es fundamental para ello. Esto es especialmente importante en objetos matemáticos de difícil conceptualización, como son los intervalos de la recta real en enseñanza secundaria. Este artículo presenta una metodología teórica para analizar el grado de congruencia entre distintas representaciones de un objeto matemático, adaptando y ampliando los tres criterios dados por Duval (1999), y creando un índice de congruencia para una conversión entre representaciones de un objeto matemático. La aplicación de la metodología de análisis se ilustra para el caso concreto de los intervalos no acotados de la recta real. Asumiendo
\end{abstract}

Fecha de recepción: 26 de febrero de 2018. Fecha de aceptación: 5 de junio de 2018.

1 Profesora Asociada del Área de Didáctica de la Matemática. Facultad de Educación y Trabajo Social, Valladolid. Universidad de Valladolid, cristina.pecharroman@uva.es, orcid.org/0000-0002-5997-5748.

2 Profesor Ayudante Doctor del Área de Didáctica de la Matemática. Facultad de Educación, Segovia. Universidad de Valladolid, matias.arce@uva.es, orcid.org/0000-0002-0770-8322.

3 Profesora Ayudante Doctor del Área de Didáctica de la Matemática. Facultad de Educación, Soria. Universidad de Valladolid, laura.conejo@uva.es, orcid.org/0000-0002-1419-1958.

4 Catedrático de Universidad del Área de Didáctica de la Matemática. Facultad de Educación y Trabajo Social, Valladolid. Universidad de Valladolid, tomas.ortega@uva.es, orcid.org/0000-0002-1116-8226. 
que la menor congruencia genera dificultades de aprendizaje inherentes al objeto, utilizamos los resultados obtenidos para proporcionar una serie de reflexiones y recomendaciones ligadas al aprendizaje de los intervalos no acotados de la recta real.

Palabras clave: intervalo; recta real; representaciones; congruencia; dificultad de una conversión.

\begin{abstract}
According to Duval $(1999,2006)$, it is considered that the apprehension of a concept involves the comprehensive and natural use of its representations, and that the ability to make conversions between representations is critical for it. This issue is particularly important in mathematical objects with a complex conceptualization, such as the intervals of the real line in secondary education. This article presents a theoretical methodology to analyze the degree of congruence between several representations of a mathematical object. In this methodology, we adapt and expand the three criteria given by Duval (1999), creating a congruence rate of a conversion between representations of a mathematical object. The application of the methodology of analysis is illustrated through the case of the unbounded intervals of the real line. Assuming that less congruence between representations produces learning difficulties inherent in the object, we use the results obtained to propose some reflections and recommendations on the learning of unbounded intervals of the real line.
\end{abstract}

Keywords: interval; real line; representations; congruence, difficulty of a conversion.

\title{
1. INTRODUCCIÓN Y OBJETIVOS
}

Las matemáticas son una asignatura fundamental en la enseñanza secundaria pero, también, una de las consideradas más difíciles. A diferencia de otras disciplinas, se trabaja con objetos propios de un dominio conceptual (Rico, 2009). Así, es fundamental conocer los signos (representaciones) que nos permiten hacer presentes los objetos y poder razonar sobre ellos. La representación de objetos es un proceso matemático fundamental destacado por múltiples autores (Berciano, Ortega y Puerta, 2015; Castro y Castro, 1997; Janvier, 1987; Kaput, 
1991), así como por el National Council of Teachers of Mathematics estadounidense (NCTM, 2000), que lo considera uno de los cinco procesos matemáticos clave (junto con la comunicación, el razonamiento y prueba, la resolución de problemas y las conexiones entre conceptos), o por el marco teórico de PISA de resolución de problemas en matemáticas de la Organización para la Cooperación y el Desarrollo Económico (OECD, 2012).

Esta necesidad e importancia nos remite a las ideas de Duval $(1999,2006)$, que postula una teoría sobre el aprendizaje de los conceptos matemáticos a través de sus representaciones y la coordinación entre ellas. Este autor señala la no-congruencia entre las representaciones de un concepto como una dificultad innata del propio concepto al llevar a cabo estas coordinaciones. Por tanto, el estudio de la congruencia entre representaciones puede ayudar a entender mejor el aprendizaje de conceptos matemáticos, especialmente cuando su conceptualización es compleja, y aumenta la necesidad de aproximarse al concepto a través de sus diferentes representaciones.

Un ejemplo de concepto matemático de difícil conceptualización en enseñanza secundaria, por el carácter topológico de su definición, es el de intervalo de la recta real. En España, el currículo de las últimas leyes educativas en los grados 10 y 11 (Ministerio de Educación y Cultura, 2007a, 2007b; Ministerio de Educación, Cultura y Deporte, 2015) indica la necesidad de conocer el significado del concepto de intervalo, conocer y reconocer (identificar y discriminar) diferentes tipos de intervalos, así como diferentes formas de expresarlos o representarlos. Su importancia radica en que facilitan la comprensión y el uso de la recta real, y en que son necesarios para desarrollar conceptos y procedimientos propios del álgebra y del análisis matemático, como pueden ser la resolución de inecuaciones, el estudio y expresión de propiedades en funciones reales de variable real o la interpretación de teoremas matemáticos.

Sin embargo, no hemos encontrado investigaciones sobre los procesos de enseñanza-aprendizaje de intervalos de la recta real. Sí hay investigaciones que han tratado la representación de los números reales en la recta real, estudiando las diferentes concepciones de la misma que pueden tener los estudiantes (Montoro, Cifuentes, Salva y Bianchi, 2017), así como los conflictos que pueden surgir en la representación de dichos números (Scaglia y Coriat, 2003). Los intervalos de la recta real son conjuntos infinitos, siendo la noción de infinito algo esencial para comprender muchos conceptos matemáticos, pero en el aprendizaje del infinito aparecen varios modelos intuitivos y metáforas lingüísticas que pueden complicarlo (Belmonte y Sierra, 2011). En el caso 
particular de los intervalos no acotados, además, aparece un proceso infinito ligado a la no acotación.

En nuestra experiencia como docentes hemos observado la existencia de bastantes dificultades en los estudiantes al trabajar con los intervalos de la recta real y, en particular, con sus representaciones y con las conversiones de unas representaciones a otras. En educación matemática sí que existen investigaciones que versan sobre realización de conversiones entre representaciones por parte de los alumnos (por ejemplo, Adu-Gyamfi y Bossé, 2014; Elia, Panaoura, Eracleous y Gagatsis, 2007). Un caso particularmente tratado ha sido la conversión de expresiones verbales a ecuaciones, donde aparece el llamado "error de inversión" (Clement, 1982). Alumnos que escriben algebraicamente "hay seis veces más estudiantes (E) que profesores (P)" en la forma $6 \mathrm{E}=\mathrm{P}$ cometen un error de inversión. Éste ha tenido diferentes explicaciones e interpretaciones, pero algunas investigaciones recientes (González-Calero, Arnau y Laserna-Belenguer, 2015; Laserna-Belenguer, Arnau y González-Calero, 2014) refuerzan la explicación del error provocado por la coincidencia en el orden de las palabras (traducción sintáctica palabra por palabra), derivado de una falta de congruencia en la conversión.

El estudio del grado de congruencia entre las representaciones de un concepto puede servir de apoyo para aportar información útil que informe sobre su proceso de aprendizaje y la detección de posibles dificultades ligadas a las conversiones entre representaciones. Así, este trabajo consta de tres objetivos principales:

- Establecer una metodología teórica para analizar la congruencia de las representaciones de los objetos matemáticos.

- Ilustrar la aplicación de la metodología para analizar la congruencia de las representaciones de los intervalos no acotados de la recta real, y comparar las conversiones entre dichas representaciones según su nivel de congruencia.

- Formular una serie de reflexiones y recomendaciones didácticas basadas en el estudio de la congruencia, para favorecer el aprendizaje de este concepto en las aulas de enseñanza secundaria.

En el siguiente apartado explicamos el marco teórico de referencia del estudio. En el tercer apartado detallamos la metodología teórica de análisis de la congruencia, concretando su aplicación para el concepto de intervalo no acotado 
de la recta real. Los resultados son utilizados en el último apartado para proponer varias recomendaciones didácticas y aspectos a tener presentes en el aprendizaje del concepto de intervalo no acotado de la recta real.

\section{MARCO TEÓRICO}

Partimos de la asunción de que los objetos matemáticos, al ser objetos propios de un dominio conceptual, no tienen existencia material (Duval, 1999, 2006; Rico, 2009). Estos objetos, sin embargo, sí que atesoran una existencia real, al ser producidos y percibidos a través de la razón (Pecharromán, 2013). Por no ser objetos físicamente accesibles, es necesario usar signos que permitan hacerlos presentes y poder comunicar ideas sobre ellos. Esos signos son denominados generalmente como representaciones, que se dotan de sentido dentro de un sistema de significados y relaciones (Rico, 2009).

Así, desde esta visión, el aprendizaje de un concepto matemático está muy ligado al dominio de sus representaciones. Según Duval (1999: 30), un estudio sobre el aprendizaje de un concepto debe tener en cuenta la diversidad de registros de representación, su coordinación y la diferenciación entre representante y representado como fundamento de la comprensión. Autores como Duval $(1999,2006)$ o Socas $(2007)$ plantean el acceso a los conceptos matemáticos a través de determinadas acciones sobre sus representaciones: reconocimiento de elementos de un sistema de representación, transformaciones dentro del mismo, conversiones de un sistema de representación a otro y coordinaciones entre representaciones en diferentes sistemas. En todo caso, se sitúa el umbral del aprendizaje de un concepto en la coordinación espontánea de al menos dos representaciones del mismo.

Sin embargo, Duval (1999) observa que existen dificultades para la coordinación entre representaciones y, también, encapsulamientos en algunas de ellas. Este autor define la congruencia entre dos representaciones como el grado de correspondencia existente entre una y otra. La falta de congruencia entre los registros de representación y la sintaxis representacional propia de cada registro hace que esta correspondencia no siempre pueda manifestarse o sea evidente. En ese sentido, Duval (1999) indica que la dificultad de conversión de una representación depende del grado de no-congruencia entre la representación de salida y la representación de llegada, siendo mayor cuanto menor sea la 
congruencia existente. Este autor considera tres criterios para analizar la congruencia (pp. 50-51), que denotaremos como CR1, CR2 y CR3:

- CR1: Correspondencia "semántica" entre las unidades significantes (de ahora en adelante, US) que constituyen las representaciones, entendiendo por US todas aquellas unidades dependientes del "léxico" de un registro de representación que no admiten descomposición en otras más simples. En el siguiente ejemplo existe correspondencia semántica entre US: "el conjunto de puntos cuya ordenada es superior a la abscisa" e " $y>x$ ". 0 siendo más precisos, $\{(x, y) \mid y>x\}$.

- CR2: Univocidad "semántica" terminal: a cada US de la representación de salida le corresponde una única US en el registro de llegada. Un ejemplo en el que no se cumple este criterio sería la US "positivo" en el registro verbal, al que le corresponden dos US en el registro algebraico ">" y "0".

- CR3: Mismo orden de aprehensión de las US en correspondencia "semántica". Se analiza si en representaciones en las que existe un mismo número de US, y en correspondencia semántica, estas US son aprehendidas (presentadas) en un mismo orden. En el caso de los intervalos, el orden de aprehensión se identifica desde el orden de lectura, de izquierda a derecha.

Hay distintos grados de congruencia, según se cumplan o no los criterios enunciados. La falta de congruencia y la consecuente dificultad de conversión son mayores en el siguiente ejemplo: "el conjunto de puntos cuya abscisa y ordenada tengan el mismo signo" y $x \cdot y>0$, (Duval, 2006: 113). No hay correspondencia entre las US, no hay univocidad semántica terminal (puesto que a la expresión "tengan el mismo signo" o "positivo" le corresponde ">0") y las US de las representaciones no se manifiestan en el mismo orden en cada registro, ya que la conversión inversa se podría realizar: "la abscisa por la ordenada es mayor que cero".

Estos tres criterios serán la base de la metodología teórica para analizar la congruencia entre representaciones que presentamos en el siguiente apartado. Los resultados de su aplicación nos aportarán información sobre la dificultad de conversión, lo cual puede traducirse en la existencia de un mayor número de errores durante el desarrollo del aprendizaje por parte de los alumnos (Rico, 1998). Esta hipótesis que relaciona la falta de congruencia con una mayor dificultad nos sirve de apoyo para proponer una serie de consideraciones didácticas orientadas hacia el diseño de secuencias de enseñanza más adecuadas, en este caso, para el aprendizaje de los intervalos no acotados de R. 


\section{METODOLOGÍA TEÓRICA DE ANÁLISIS PARA ESTUDIAR LA CONGRUENCIA ENTRE LAS REPRESENTACIONES: EL CASO CONCRETO DE LOS INTERVALOS NO ACOTADOS}

Partiendo de una serie de consideraciones previas ligadas al análisis, sigue una propuesta metodológica para analizar de forma teórica la congruencia entre sistemas de representación de un objeto matemático, y obtener un índice de congruencia entre representaciones en los diferentes sistemas semióticos. La metodología se ilustra a través del desarrollo de la misma en el caso de los intervalos no acotados de la recta real, y considerando cuatro representaciones de los mismos: verbal, gráfica, numérica y algebraica.

\subsection{CONSIDERACIONES PREVIAS LIGADAS AL ANÁLISIS DE LA CONGRUENCIA}

Presentamos varias consideraciones previas, necesarias para aplicar la metodología de análisis de la congruencia.

Los alumnos deben tener una serie de conocimientos previos para poder llevar a cabo el aprendizaje. La ausencia o el desarrollo parcial de estos conocimientos previos puede ser una fuente importante de dificultades para el propio proceso de representación, al ser un prerrequisito para ello. No obstante, al tratarse de un estudio teórico de análisis de la congruencia, asumimos un hipotético dominio de estos conocimientos previos. En el ejemplo de los intervalos de la recta real, los conocimientos previos necesarios son: saber representar números reales en dicha recta real, saber posicionar el $\infty^{5}(+\infty$ y $-\infty)$, conocer los símbolos de desigualdad y la relación de orden que implica, conocer de forma intuitiva o experimental la propiedad de densidad de la recta real y tener conocimientos mínimos sobre los registros semióticos en los que va a ser representado el concepto.

Deben analizarse los signos matemáticos que configuran las representaciones del objeto matemático, y su significado. La Tabla 1 presenta los asociados al concepto de intervalo de la recta real.

\footnotetext{
5 No haremos distinción entre $\infty$ y $+\infty$ ni entre infinito y más infinito. Solo se escribirán $+\infty$ y más infinito. Asimismo, se considera que $+\infty$ y $-\infty$ son sendas unidades de significado, lo mismo que, por ejemplo, $+3 y-3$
} 
Tabla 1. Correspondencia de signos con el mismo significado en los diferentes registros.

\begin{tabular}{|c|c|c|c|c|}
\hline Significado & Verbal & Gráfico & Numérico & Algebraico \\
\hline $\begin{array}{l}\text { El número (extremo del } \\
\text { intervalo) no forma } \\
\text { parte del mismo }\end{array}$ & Abierto & $\begin{array}{l}\text { Se lee: punto } \\
\text { vacío. }\end{array}$ & $\begin{array}{c}(,) \\
\text { Se lee: } \\
\text { paréntesis. }\end{array}$ & $\begin{array}{c}<y> \\
\text { Desigualdades: } \\
\text { "menor que" y "mayor } \\
\text { que" }\end{array}$ \\
\hline $\begin{array}{l}\text { El número (extremo del } \\
\text { intervalo) forma parte } \\
\text { del mismo }\end{array}$ & Cerrado & $\begin{array}{l}\text { Se lee: punto } \\
\text { relleno. }\end{array}$ & $\begin{array}{c}{[,]} \\
\text { Se lee: } \\
\text { corchete. }\end{array}$ & $\begin{array}{c}\leq y \geq \\
\text { Desigualdades: } \\
\text { "menor o igual que" } \\
\text { y "mayor o igual que" }\end{array}$ \\
\hline $\begin{array}{l}\text { Números en el intervalo } \\
\text { (acotado o no) }\end{array}$ & Números & $\begin{array}{l}\text { Segmento o } \\
\text { semirrecta }\end{array}$ & (No hay) & $x$ \\
\hline Infinitud de la recta real & $\begin{array}{l}\text { Infinito y } \\
\text { menos infinito }\end{array}$ & Flecha & $+\infty y-\infty$ & (No hay) \\
\hline
\end{tabular}

La lectura usual de las representaciones y de los signos que las configuran se realiza de izquierda a derecha, según el flujo de lectura. En los intervalos, se corresponde con la relación de orden usual de la recta real. No obstante, tanto el registro verbal como el algebraico admiten distintas representaciones. Esta posibilidad ha generado la necesidad de añadir un criterio de congruencia previo a los tres ya establecidos por Duval. Este criterio, que denotamos por CR0, hace mención a la representación global, y es el siguiente:

- CR0: Univocidad representacional: A la representación en el registro de partida le corresponde una única representación en el registro de llegada. No existiría univocidad si una representación de partida tuviera asociadas dos o más representaciones posibles en el registro de llegada.

En el caso de los intervalos, los símbolos de desigualdad que configuran la representación algebraica tienen doble función-uso y permiten doble lectura.

- La doble función del símbolo de desigualdad (representar a la vez lo que es menor y lo que es mayor) hace que puedan utilizarse dos representaciones algebraicas para un mismo intervalo, que difieren en el orden de colocación 
de los elementos constituyentes. Por ejemplo, puede escribirse $e^{6}$ tanto $x>$ a como $a<x$ para representar un intervalo no acotado. Lo mismo con $x \geq a \mathrm{y} a \leq x$.

- La posibilidad de doble lectura del símbolo de desigualdad motiva que haya al menos dos representaciones verbales para cada representación algebraica, teniendo como referencia el símbolo de desigualdad. El símbolo " $<$ " se lee "menor" si se interpreta desde la izquierda, es decir, siguiendo el flujo de lectura o el orden usual de la recta real; y se lee "mayor" si es interpretado desde la derecha. Análogamente sucede con ">". Además, la representación verbal admite diferentes representaciones, en función de los elementos lingüísticos elegidos para su configuración o del orden en que sean configurados. Por ejemplo, puede comenzarse haciendo referencia al elemento genérico ("números") o ser meramente descriptiva, limitándose a nombrar los signos que aparecen en la representación.

En este sentido, hipotetizamos sobre la existencia de dos criterios diferentes que pueden utilizarse para obtener la representación de un intervalo en algunos sistemas de representación:

- Orden real (de ahora en adelante, OR): Se presentan los símbolos coincidiendo con el orden de recorrido de la recta real (asociado algebraicamente al uso del símbolo <), que también coincide con el flujo de lectura. Este criterio es el que mejor se corresponde con la búsqueda de la congruencia de la conversión y con la acción descriptiva de la representación. En caso contrario, escribiremos "NoOR".

- Orden lingüístico (de ahora en adelante, OL): La expresión utilizada prioriza el significado del concepto frente a la disposición de los elementos que configuran la representación. Se presenta en primer lugar el elemento genérico del intervalo correspondiente: "los números, x" que lo constituyen. Un ejemplo en el registro verbal: "Números mayores que a", frente a la que seguiría el OR y sería meramente descriptiva: "a menor que los números del intervalo". Un ejemplo en el registro algebraico: " $x>a$ ", frente al $O R$ " $a<x$ ”.

6 Por simplicidad, consideraremos en la representación algebraica la escritura de la condición que cumplen los puntos del intervalo (en este caso, $x<a$ ), en lugar de la escritura conjuntista completa $(\{x \in \mathbb{R} \mid x<a\})$, dado que la escritura de la condición es la parte variable en la representación de diferentes intervalos. 
Para aplicar la metodología es necesario disponer de representaciones en varios registros de un mismo objeto matemático. En la Tabla 2 presentamos los casos de intervalos abiertos en la recta real, tanto acotados como no acotados, y algunas posibilidades de expresión en los registros gráfico, numérico, verbal y algebraico. En el caso del registro verbal hemos considerado dos alternativas, en función de si se indica antes el extremo del intervalo o los números que lo conforman, pero utilizando dos representantes que sintetizan la mayoría de expresiones que pueden utilizarse en las aulas. Por ejemplo, utilizamos por simplicidad "a menor que números" como expresión verbal equivalente a otras como "a es menor que todos los números del intervalo", que pueden ser más habituales en el lenguaje pero que no aumentan el número de unidades significantes con respecto a la expresión seleccionada.

Las representaciones serían análogas en intervalos cerrados.

Tabla 2. Algunas posibilidades de expresión de un intervalo en los distintos registros.

\begin{tabular}{|c|c|c|c|}
\hline Registro & Intervalo acotado & $\begin{array}{l}\text { Intervalo no acotado } \\
\text { por la derecha }\end{array}$ & $\begin{array}{c}\text { Intervalo no acotado } \\
\text { por la izquierda }\end{array}$ \\
\hline Gráfico & $b_{0}$ & $a^{a}$ & $\mathrm{a}^{a}$ \\
\hline Numérico & $(a, b)$ & $(\mathrm{a},+\infty)$ & $(-\infty, a)$ \\
\hline \multirow[t]{2}{*}{ Verbal } & 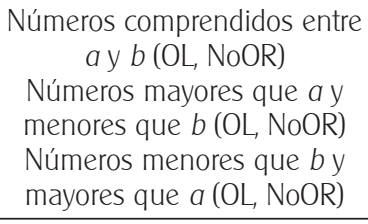 & $\begin{array}{c}\text { Números mayores que } \\
\text { a (OL, NoOR) }\end{array}$ & $\begin{array}{c}\text { Números menores que } \\
\qquad a(\mathrm{OL}, \mathrm{OR})\end{array}$ \\
\hline & $\begin{array}{c}\text { a menor que números } \\
\text { menores que } b \text { (NoOL, OR) } \\
b \text { mayor que números } \\
\text { mayores que } a \text { (NoOL, NoOR) }\end{array}$ & $\begin{array}{c}\text { a menor que números } \\
\text { (NoOL, OR) }\end{array}$ & $\begin{array}{l}\text { a mayor que números } \\
\text { (NoOL, NoOR) }\end{array}$ \\
\hline \multirow{2}{*}{ Algebraico } & $\mathrm{a}<\mathrm{x}<\mathrm{b}($ NoOL, OR) & $x>a(O L, N o O R)$ & $\mathrm{x}<\mathrm{a}(\mathrm{OL}, \mathrm{OR})$ \\
\hline & $b>x>a(N o O L, N o O R)$ & $\mathrm{a}<\mathrm{x}(\mathrm{NoOL}, \mathrm{OR})$ & $\mathrm{a}>\mathrm{x}(\mathrm{NoOL}, \mathrm{NoOR})$ \\
\hline
\end{tabular}

El último paso previo necesario es la elección de las unidades significantes que componen las representaciones del objeto matemático, a partir de las cuales poder estudiar la congruencia. En el ejemplo de intervalos se han considerado cinco US: 
- US1: Los números genéricos que configuran el intervalo (por ejemplo: "x", intervalo no acotado que se marca, "números").

- US2: Los números que limitan el intervalo (extremos).

- US3: El más infinito o el menos infinito (para el caso de los intervalos no acotados).

- US4: Los elementos que indican la inclusión o no de los números que limitan el intervalo (extremos).

- US5: Indicadores de la posición de unos elementos respecto a otros, dada por la posición de los objetos en la representación en los registros gráfico y numérico, por los símbolos de desigualdad en el algebraico y las expresiones verbales que los ubican en el verbal.

Para el criterio CR1 (correspondencia semántica entre las unidades significantes), tomaremos como referencia el porcentaje de las US en las cuales existe correspondencia con respecto al total. Asimismo, para CR2 consideramos que cuando a una US de la representación de partida no le corresponda ninguna US de la de llegada, no hay univocidad semántica terminal.

\subsection{DeSARROLLO DE LA METOdOLOGÍA: ANÁLISIS TEÓRICO DE LA CONGRUENCIA PARA CADA CONVERSIÓN}

Se ilustra el desarrollo de la metodología teórica para el ejemplo de los intervalos de la recta real, pero el desarrollo es aplicable a otros objetos matemáticos. Los cuatro registros de representación aquí considerados (gráfico, numérico, algebraico y verbal) dan lugar a seis conversiones. Mostramos el análisis de cada una, utilizando la siguiente estructura de presentación: se recogen en una tabla las representaciones en cada registro, considerando intervalos no acotados, tanto por la derecha como por la izquierda. Le sigue el estudio de la congruencia entre las representaciones situadas en una misma fila de la tabla, de acuerdo con los criterios de congruencia fijados. El análisis realizado se circunscribe a intervalos no acotados, en los que además de ser subconjuntos infinitos de la recta real, existen procesos infinitos ligados a esa falta de acotación (bien por la izquierda o por la derecha) y la simbología propia del infinito, que pueden suponer una complicación añadida con respecto a los intervalos acotados. 


\section{Conversión 1: Gráfico-Numérica (CG-N)}

Tabla 3. Representaciones de intervalos no acotados en los registros gráfico y numérico.

\begin{tabular}{|c|c|}
\hline Registro gráfico & Registro numérico \\
\hline$a^{a}$ & $(a,+\infty)$ \\
\hline$\sim^{a}$ & $(-\infty, \mathrm{a})$ \\
\hline
\end{tabular}

Análisis de la congruencia entre los registros gráfico y numérico, cuyas representaciones están en la Tabla 3:

CR0 (Univocidad representacional): Sí existe, a cada representación en el registro de partida le corresponde una única representación en el registro de llegada.

CR1 (Correspondencia semántica entre US): No hay correspondencia total entre las unidades. Sí que existe para la US2, US3 y la US4. Sin embargo, en la representación numérica falta la expresión de los números, "x", que configuran el intervalo (US1) y, por tanto, la indicación sobre su ubicación (US5). En suma, hay $60 \%$ de correspondencia semántica.

CR2 (Univocidad "semántica" terminal): No hay univocidad, pues a un elemento de la representación gráfica (la semirrecta marcada, que expresa los números que incluye el intervalo) no le corresponde ningún elemento de la representación numérica.

CR3 (Orden de aprehensión): Se mantiene el orden en el que aparecen los elementos de la representación en correspondencia semántica en ambos registros.

\section{Conversión 2: Gráfico-Algebraica (CG-A)}

Tabla 4. Representaciones de intervalos no acotados en los registros gráfico y algebraico.

\begin{tabular}{|c|c|}
\hline Registro gráfico & Registro algebraico \\
\hline \multirow{2}{*}{$a^{a}$} & x>a (OL, NoOR) \\
\hline & $\mathrm{a}<\mathrm{x}$ (NoOL, OR) \\
\hline \multirow{2}{*}{ a } & $\mathrm{x}<\mathrm{a}(\mathrm{OL}, \mathrm{OR})$ \\
\hline & $\mathrm{a}>\mathrm{x}(\mathrm{NoOL}, \mathrm{NoOR})$ \\
\hline
\end{tabular}


Análisis de la congruencia entre los registros gráfico y algebraico, cuyas representaciones se recogen en la Tabla 4:

CR0 (Univocidad representacional): No existe, porque cada representación en el registro gráfico admite dos representaciones en el registro algebraico.

CR1 (Correspondencia semántica): Existe correspondencia entre los números que configuran el intervalo (US1), los números que lo limitan (US2), la indicación de su inclusión o no (US4) y la indicación de la posición de unos elementos respecto a otros (US5). En cambio, la flecha que indica la infinitud de la semirrecta no tiene US correspondiente en la representación algebraica (US3). Por tanto, no hay correspondencia total entre los elementos que configuran las representaciones ( $80 \%$ de correspondencia semántica).

CR2 (Univocidad "semántica" terminal): No existe, porque el símbolo desigualdad indica tanto la exclusión del extremo del intervalo no acotado (US4, punto vacío del registro gráfico) como la posición de los números que configuran el intervalo respecto a ese extremo (US5, semirrecta marcada en el registro gráfico).

CR3 (Orden de aprehensión): En cada caso, una representación algebraica mantiene el orden en el cual aparecen los elementos en la representación gráfica, que coincide con el orden de lectura (OR). La otra representación no mantiene ese orden.

\section{Conversión 3: Gráfico-Verbal (CG-V)}

Tabla 5. Representaciones de intervalos no acotados en los registros gráfico y verbal.

\begin{tabular}{c|c}
\hline Registro gráfico & Registro verbal \\
\hline \multirow{2}{*}{\begin{tabular}{c}
\multirow{2}{*}{} \\
\cline { 2 - 2 }
\end{tabular}} & Números mayores que $a(\mathrm{OL}, \mathrm{NoOR})$ \\
\cline { 2 - 2 } & a menor que números (NoOL, OR) \\
\hline
\end{tabular}

Análisis de la congruencia entre los registros gráfico y verbal, cuyas representaciones aparecen en la Tabla 5:

CR0 (Univocidad representacional): No existe, ya que a cada representación en el registro gráfico le corresponden dos representaciones, al menos, en el registro verbal. 
CR1 (Correspondencia semántica): Hay correspondencia entre los números que configuran el intervalo (US1), los números que lo limitan (US2), la indicación de su inclusión o no (US4) y la indicación de la posición de unos elementos respecto a otros (US5). Sin embargo, la flecha que marca gráficamente la infinitud de la semirrecta no tiene correspondencia en la representación verbal (US3). Así, hay una correspondencia semántica de 80\%.

CR2 (Univocidad "semántica" terminal): No, porque a las palabras "mayores" y "menores" les corresponden tanto la exclusión del extremo del intervalo no acotado (US4, punto vacío) como la posición de los números que configuran el intervalo respecto a ese extremo (US5).

CR3 (Orden de aprehensión): En cada caso, una de las representaciones verbales mantiene el orden de aparición de los elementos en la representación gráfica (coincidente con el orden de lectura, OR). La otra no mantiene ese orden.

\section{Conversión 4: Numérica-Verbal (CN-V)}

Tabla 6. Representaciones de intervalos no acotados en los registros numérico y verbal.

\begin{tabular}{c|c}
\hline Registro numérico & Registro verbal \\
\hline \multirow{2}{*}{$(a,+\infty)$} & Números mayores que $a(\mathrm{OL}, \mathrm{NoOR})$ \\
\cline { 2 - 2 }$(-\infty, \mathrm{a})$ & $a$ menor que números (NoOL, OR) \\
\cline { 2 - 2 } & Números menores que $a(\mathrm{OL}, \mathrm{OR})$ \\
\hline \multirow{2}{*}{$(\mathrm{a}$ mayor que números (NoOL, NoOR) } \\
\hline
\end{tabular}

Análisis de la congruencia entre los registros numérico y verbal, cuyas representaciones se recogen en la Tabla 6:

CR0 (Univocidad representacional): No existe, ya que a cada representación en el registro numérico le corresponden dos representaciones, al menos, en el registro verbal.

CR1 (Correspondencia semántica): No existe correspondencia en todas las US que configuran las representaciones. Sí que sucede para la US2 y la US4, pero en la representación numérica faltan los números genéricos que configuran el intervalo y su posición (US1 y US5). Además, en las representaciones verbales consideradas falta el elemento infinito (US3). Así, hay 40\% de correspondencia semántica. 
CR2 (Univocidad "semántica" terminal): No hay univocidad, pues hay elementos en ambas representaciones a los que no les corresponde ningún elemento

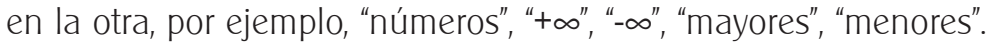

CR3 (Orden de aprehensión): Ninguna de las representaciones verbales es estrictamente descriptiva (hay elementos de la representación numérica que no se mencionan, y son mencionados otros no presentes). Para decidir sobre el orden de aprehensión, nos fijamos en que las US que componen la representación numérica y la verbal estén colocadas, respectivamente, siguiendo el mismo criterio de orden, en este caso el OR. De las dos representaciones verbales derivadas de cada una de las representaciones numéricas, una sigue el OR y otra no.

\section{Conversión 5: Numérica-Algebraica (CN-A)}

Tabla 7. Representaciones de intervalos no acotados en los registros numérico y algebraico.

\begin{tabular}{c|c}
\hline Registro numérico & Registro algebraico \\
\hline \multirow{2}{*}{$(a,+\infty)$} & $\mathrm{x}>\mathrm{a}(\mathrm{OL}, \mathrm{NoOR})$ \\
\cline { 2 - 2 }$(-\infty, \mathrm{a})$ & $\mathrm{a}<\mathrm{x}(\mathrm{NoOL}, \mathrm{OR})$ \\
\hline \multirow{2}{*}{$(\mathrm{x}<\mathrm{a}(\mathrm{OL}, \mathrm{OR})$} \\
\hline
\end{tabular}

Análisis de la congruencia entre los registros numérico y algebraico, cuyas representaciones aparecen en la Tabla 7:

CR0 (Univocidad representacional): No existe, porque cada representación en el registro numérico admite dos representaciones en el registro algebraico.

CR1 (Correspondencia semántica): Únicamente existe correspondencia para las US2 y US4 (número extremo del intervalo e indicación de su inclusión o no). En la representación numérica faltan los números que configuran el intervalo y la indicación de su posición (US1 y US5), mientras que en las representaciones algebraicas falta el elemento infinito (US3). La correspondencia semántica es de 40\%.

CR2 (Univocidad "semántica" terminal): No hay univocidad, porque al símbolo de desigualdad en el registro algebraico le corresponde tanto indicar la exclusión del extremo del intervalo no acotado (US4, paréntesis en el registro numérico) 
como la posición de los números que configuran el intervalo respecto a ese extremo (US5).

CR3 (Orden de aprehensión): Como en la conversión anterior, las US que componen la representación numérica y la algebraica pueden estar colocadas siguiendo el OR o no. De las dos representaciones algebraicas derivadas de cada representación numérica, una sigue el OR y otra no.

\section{Conversión 6: Algebraica-Verbal (CA-V)}

Tabla 8. Representaciones de intervalos no acotados en los registros algebraico y verbal.

\begin{tabular}{|c|c|}
\hline Registro algebraico & Registro verbal \\
\hline \multirow{2}{*}{$\mathrm{x}>\mathrm{a}(\mathrm{OL}, \mathrm{NoOR})$} & Números mayores que a (OL, NoOR) \\
\hline & a menor que números (NoOL, OR) \\
\hline \multirow{2}{*}{$\mathrm{a}<\mathrm{x}(\mathrm{NoOL}, \mathrm{OR})$} & Números mayores que a (OL, NoOR) \\
\hline & $a$ menor que números (NoOL, OR) \\
\hline \multirow{2}{*}{$x<a(O L, O R)$} & Números menores que a (OL, OR) \\
\hline & a mayor que números (NoOL, NoOR) \\
\hline \multirow{2}{*}{$\mathrm{a}>\mathrm{x}(\mathrm{NoOL}, \mathrm{NoOR})$} & Números menores que a (OL, OR) \\
\hline & a mayor que números (NoOL, NoOR) \\
\hline
\end{tabular}

Análisis de la congruencia entre los registros algebraico y verbal, cuyas representaciones están en la Tabla 8:

CR0 (Univocidad representacional): No existe, porque cada una de las representaciones en el registro algebraico admite al menos dos representaciones en el registro verbal. Además, a cada una de las representaciones en el registro verbal le corresponden dos representaciones en el registro algebraico.

CR1 (Correspondencia semántica): Hay correspondencia entre todas las US que configuran las representaciones: los números que configuran el intervalo (US1), los números que lo limitan, la inclusión o no de estos y la indicación de la posición de unos respecto a otros (US2, US4 y US5), y la no presencia del elemento infinito (US3). Hay 100\% de correspondencia semántica.

CR2 (Univocidad "semántica" terminal): No existe univocidad, porque al símbolo de desigualdad (en el registro algebraico) y a las palabras "mayores" y 
"menores" (en el registro verbal) le corresponden tanto la exclusión del extremo de la semirrecta (US4) como la indicación de la posición de los números que configuran el intervalo respecto a ese extremo (US5). Por ejemplo, a la US4 en el registro algebraico " $<$ " le corresponde el signo "menores" en el registro verbal, que tiene dos unidades de significado (US4 y US5). Recíprocamente, a la US4 en el registro verbal "menores" le corresponde el signo " $<$ ", que tiene dos unidades de significado (US4 y US5). Lo mismo sucede con los signos ">" y "mayores".

CR3 (Orden de aprehensión): Dado que las representaciones verbales no son estrictamente descriptivas, estas y las representaciones algebraicas que conservan el orden de aprehensión son aquellas que se han construido manteniendo en ambos casos bien el OR o bien el NoOR.

\subsection{OBTENCIÓN DEL ÍNDICE DE CONGRUENCIA ENTRE REPRESENTACIONES EN LOS DIFERENTES SISTEMAS SEMIÓTICOS}

Las Tablas 9 y 10 resumen el análisis teórico realizado. La primera columna de cada tabla indica la conversión asociada, la segunda muestra si hay o no univocidad entre las representaciones (criterio CR0) y la tercera explicita las representaciones involucradas (teniendo en cuenta que existe más de una conversión posible entre registros si no hay univocidad representacional). En este caso, cada conversión es analizada de forma independiente. La cuarta, quinta y sexta columna corresponden a la valoración de los criterios de congruencia de Duval (1999), aquí denotados como CR1, CR2 y CR3. La última columna contiene el índice de congruencia para cada conversión, que denotaremos como IC, entre las representaciones de distintos sistemas. Este índice se define a partir de la media aritmética de índices particulares ligados a los cuatro criterios, que son explicados a continuación de la fórmula. Utilizando la media aritmética, los cuatro criterios intervienen en el índice y se puede tanto valorar el nivel de congruencia de cada conversión como realizar comparaciones entre unas y otras:

$$
I C=\frac{I_{1}+I_{2}+I_{3}+I_{4}}{4}
$$

Siendo:

- $I_{1}$ un valor igual a $1 / n$, donde $n$ es el número de conversiones diferentes posibles asociadas a la conversión entre dos sistemas de representación (criterio CR0). Si existe univocidad representacional en una conversión (una 
representación posible dentro de cada registro), $\mathrm{I}_{1}$ valdrá 1 ( $\mathrm{n}=1$ ). Si no, el valor será inversamente proporcional al número de conversiones posibles que puedan realizarse, al existir la necesidad de conocer diversas representaciones del concepto dentro del registro para poder hacer una hipotética conversión solicitada, lo que aumenta la dificultad global ligada a la conversión.

- $I_{2}$ igual al tanto por uno del porcentaje de correspondencia de CR1.

- I I igual a 1 o 0 , según exista univocidad semántica o no en las representaciones involucradas en la conversión (criterio CR2).

- $I_{4}$ igual a 1 o 0 , según se mantenga el orden de aprehensión o no en las representaciones involucradas en la conversión (criterio CR3).

Todos los IC se presentan con fracciones con el mismo denominador, aunque no sea una fracción irreducible, para facilitar la comparación entre los diferentes IC.

A priori, podría pensarse que los valores de los IC correspondientes a los intervalos no acotados por la izquierda y por la derecha serían similares. Sin embargo, son diferentes $y$, aunque se mantiene la misma estructura, presentamos dos tablas: la Tabla 9 sobre intervalos no acotados por la derecha y la Tabla 10 sobre intervalos no acotados por la izquierda. Ambas tablas presentan las conversiones siguiendo el mismo orden considerado en las tablas parciales anteriores (Tablas 3 a 8).

Tabla 9. Tabla resumen del estudio teórico de la congruencia entre sistemas semióticos para intervalos no acotados por la derecha.

\begin{tabular}{|c|c|c|c|c|c|c|}
\hline Conversión & $\begin{array}{l}\text { CR0: } \\
\text { Univocidad } \\
\text { representa- } \\
\text { cional }\end{array}$ & $\begin{array}{l}\text { Representacio- } \\
\text { nes involucradas }\end{array}$ & $\begin{array}{l}\text { CR1: Co- } \\
\text { rrespon- } \\
\text { dencia US }\end{array}$ & $\begin{array}{l}\text { CR2: } \\
\text { Univoci- } \\
\text { dad se- } \\
\text { mántica }\end{array}$ & $\begin{array}{l}\text { CR3: Mismo } \\
\text { orden de } \\
\text { aprehensión }\end{array}$ & IC \\
\hline CG-N & Sí & $\begin{array}{l}\mathrm{G}: \underset{a^{a}}{\mathrm{a}} \stackrel{(\mathrm{a},+\infty)}{\longrightarrow}\end{array}$ & $60 \%$ & NO & Sí & $52 / 80$ \\
\hline \multirow{2}{*}{ CG-A } & \multirow{2}{*}{ NO } & 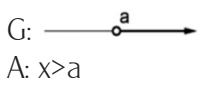 & $80 \%$ & NO & NO & $26 / 80$ \\
\hline & & 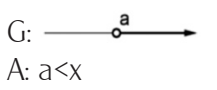 & $80 \%$ & NO & Sí & $46 / 80$ \\
\hline
\end{tabular}




\begin{tabular}{|c|c|c|c|c|c|c|}
\hline \multirow{2}{*}{ CG-V } & \multirow{2}{*}{ NO } & $\begin{array}{l}\mathrm{G}: \stackrel{\text { a }}{\mathrm{a}} \\
\mathrm{V}: \text { "Números } \\
\text { mayores que } d^{\text {" }}\end{array}$ & $80 \%$ & NO & NO & $26 / 80$ \\
\hline & & $\begin{array}{l}\mathrm{G}: \stackrel{\mathrm{a}}{\mathrm{a}} \underset{\mathrm{V}: \text { "a menor que }}{\text { números" }} \\
\end{array}$ & $80 \%$ & NO & Sí & $46 / 80$ \\
\hline \multirow{2}{*}{$\mathrm{CN}-\mathrm{V}$} & \multirow{2}{*}{ NO } & $\begin{array}{l}\mathrm{N}:(\mathrm{a},+\infty) \\
\mathrm{V}: \text { "Números } \\
\text { mayores que } d^{\prime \prime}\end{array}$ & $40 \%$ & NO & NO & $18 / 80$ \\
\hline & & $\begin{array}{l}\mathrm{N}:(\mathrm{a},+\infty) \\
\mathrm{V}: \text { " } a \text { menor que } \\
\text { números" }\end{array}$ & $40 \%$ & NO & Sí & $38 / 80$ \\
\hline \multirow{2}{*}{ CN-A } & \multirow{2}{*}{ NO } & $\begin{array}{l}N:(a,+\infty) \\
A: x>a\end{array}$ & $40 \%$ & NO & NO & $18 / 80$ \\
\hline & & $\begin{array}{l}N:(a,+\infty) \\
A: a<x\end{array}$ & $40 \%$ & NO & Sí & $38 / 80$ \\
\hline \multirow{4}{*}{ CA-V } & \multirow{4}{*}{ NO } & $\begin{array}{l}\text { A: } x>a \\
\text { V: "Números } \\
\text { mayores que } d^{\prime \prime}\end{array}$ & $100 \%$ & NO & Sí & $45 / 80$ \\
\hline & & $\begin{array}{l}\mathrm{A}: \mathrm{x}>\mathrm{a} \\
\mathrm{V}: \text { " } a \text { menor que } \\
\text { números" }\end{array}$ & $100 \%$ & NO & NO & $25 / 80$ \\
\hline & & $\begin{array}{l}\text { A: } a<x \\
\text { V: "Números } \\
\text { mayores que } d^{\prime \prime}\end{array}$ & $100 \%$ & NO & NO & $25 / 80$ \\
\hline & & $\begin{array}{l}\text { A: } a<x \\
\text { V: "a menor que } \\
\text { números" }\end{array}$ & $100 \%$ & NO & $\mathrm{SI}$ & $45 / 80$ \\
\hline
\end{tabular}

Tabla 10. Tabla resumen del estudio teórico de la congruencia entre sistemas semióticos para intervalos no acotados por la derecha.

\begin{tabular}{|c|c|c|c|c|c|c|}
\hline Conversión & $\begin{array}{l}\text { CR0: Univo- } \\
\text { cidad re- } \\
\text { presenta- } \\
\text { cional }\end{array}$ & $\begin{array}{l}\text { Representacio- } \\
\text { nes involucradas }\end{array}$ & $\begin{array}{l}\text { CR1: Co- } \\
\text { rrespon- } \\
\text { dencia US }\end{array}$ & $\begin{array}{l}\text { CR2: } \\
\text { Univoci- } \\
\text { dad se- } \\
\text { mántica }\end{array}$ & $\begin{array}{l}\text { CR3: Mismo } \\
\text { orden de } \\
\text { aprehensión }\end{array}$ & IC \\
\hline CG-N & sí & 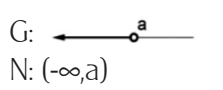 & $60 \%$ & NO & sí & $52 / 80$ \\
\hline
\end{tabular}




\begin{tabular}{|c|c|c|c|c|c|c|}
\hline \multirow{2}{*}{ CG-A } & \multirow{2}{*}{ NO } & 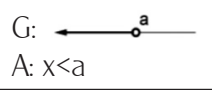 & $80 \%$ & NO & sí & $46 / 80$ \\
\hline & & $\begin{array}{l}G: \varlimsup^{a}=a\end{array}$ & $80 \%$ & NO & NO & $26 / 80$ \\
\hline \multirow{2}{*}{$C G-V$} & \multirow{2}{*}{ NO } & 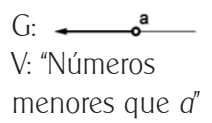 & $80 \%$ & NO & sí & $46 / 80$ \\
\hline & & $\begin{array}{l}\mathrm{G}:{ }_{\mathrm{V}:}^{\mathrm{a}} \stackrel{\mathrm{a}}{\mathrm{númayor} \text { que }} \\
\text { núm" }\end{array}$ & $80 \%$ & NO & NO & $26 / 80$ \\
\hline \multirow{2}{*}{$\mathrm{CN}-\mathrm{V}$} & \multirow{2}{*}{ NO } & $\begin{array}{l}\mathrm{N}:(-\infty, \mathrm{a}) \\
\mathrm{V}: \text { "Números } \\
\text { menores que } d^{\prime \prime}\end{array}$ & $40 \%$ & NO & sí & $38 / 80$ \\
\hline & & $\begin{array}{l}\mathrm{N}:(-\infty, \mathrm{a}) \\
\mathrm{V}: \text { "a mayor que } \\
\text { números" }\end{array}$ & $40 \%$ & NO & NO & $18 / 80$ \\
\hline \multirow{2}{*}{ CN-A } & \multirow{2}{*}{ NO } & $\begin{array}{l}N:(-\infty, a) \\
A: x<a\end{array}$ & $40 \%$ & NO & sí & $38 / 80$ \\
\hline & & $\begin{array}{l}N:(-\infty, a) \\
A: a>x\end{array}$ & $40 \%$ & NO & NO & $18 / 80$ \\
\hline \multirow{4}{*}{$C A-V$} & \multirow{4}{*}{ NO } & $\begin{array}{l}\mathrm{A}: \mathrm{x}<\mathrm{a} \\
\mathrm{V}: \text { "Números } \\
\text { menores que } d^{\prime \prime}\end{array}$ & $100 \%$ & NO & Sí & $45 / 80$ \\
\hline & & $\begin{array}{l}\mathrm{A}: \mathrm{x}<\mathrm{a} \\
\mathrm{V}: \text { "a mayor que } \\
\text { números" }\end{array}$ & $100 \%$ & NO & NO & $25 / 80$ \\
\hline & & $\begin{array}{l}\text { A: } a>x \\
V: \text { "Números } \\
\text { menores que } d \text { " }\end{array}$ & $100 \%$ & NO & NO & $25 / 80$ \\
\hline & & $\begin{array}{l}\mathrm{A}: \mathrm{a}>\mathrm{x} \\
\mathrm{V}: \text { "a mayor que } \\
\text { números" }\end{array}$ & $100 \%$ & NO & sí & $45 / 80$ \\
\hline
\end{tabular}

Observamos que en ningún caso se cumplen todos los criterios de congruencia, ya que todos los índices son menores que la unidad. En los casos sin univocidad representacional, Ios IC varían en función de cuál sea la representación utilizada dentro de un sistema semiótico, siendo los índices más altos aquellos que 
mantienen el orden de aprehensión (CR3). El índice de congruencia más alto (52/80) es el ligado a la conversión gráfica-numérica, en buena medida debido a la existencia de una única forma de representar el objeto en cada registro (univocidad representacional). Los menores índices de congruencia (18/80) se han obtenido en las conversiones numérica-verbal, por una parte, y numérica-algebraica, por otra, en aquellos casos en los cuales las representaciones no mantienen el mismo orden de aprehensión.

Como indica Duval (1999), la no congruencia entre diferentes registros de representación es motivo de dificultades de aprendizaje y de posibles errores en las producciones de los alumnos. Desde este punto de vista, los resultados del análisis teórico realizado (Tablas 9 y 10) aportan información significativa sobre el grado de dificultad de cada conversión; por tanto, conviene tener en cuenta los resultados obtenidos en el análisis de la congruencia, y la información que nos proporcionan, para el planteamiento y diseño de secuencias didácticas de enseñanza en el aula del concepto de intervalo de la recta real, en general, y de los intervalos no acotados en particular.

\section{DISCUSIÓN DE LOS RESULTADOS EN RELACIÓN CON EL APRENDIZAJE DE LOS INTERVALOS NO ACOTADOS DE LA RECTA REAL: REFLEXIONES Y RECOMENDACIONES DIDÁCTICAS}

Hay que tener en cuenta que los índices de congruencia mayores expresan que la conversión (coordinación) entre representaciones es más sencilla, a priori, que en aquellas conversiones (coordinaciones) cuyos IC sean menores. Esta relación entre congruencia y dificultad, y la relación establecida por Duval (1999) entre el aprendizaje de un concepto y la coordinación espontánea de sus representaciones en al menos dos registros, permiten establecer una hipótesis de aprendizaje de los objetos matemáticos. Así, parece natural afirmar que aquellas coordinaciones entre representaciones que presentan mayor congruencia pueden ser las coordinaciones inicialmente dominadas por los estudiantes, y contribuir al desarrollo del aprendizaje del objeto representado. Es decir, esas coordinaciones podrían facilitar que el estudiante inicie el proceso paulatino de independencia del objeto matemático de sus posibles representaciones y, efectivamente, que las representaciones del objeto sean percibidas como diferentes medios de expresión del mismo, que pueden además tener diferentes funcionalidades (Pecharromán, 2013). 
No obstante, es necesario puntualizar que la generación de representaciones de un objeto matemático, de conversiones entre ellas y ese proceso de independencia del objeto de sus posibles representaciones puede presentar en la práctica real otras dificultades, además de las directamente relacionadas con el índice de congruencia entre representaciones. Como indicábamos al inicio del tercer apartado, la ausencia o el desarrollo insuficiente de los conocimientos previos que se consideren prerrequisito indispensable influirán en los procesos de generación de representaciones y conversiones, y serán fuente de dificultades y errores. Además, cada objeto matemático puede tener características particulares que lo definan en su complejidad, y que pueden verse reflejadas en la necesidad de una mayor cantidad de conocimientos previos, o de conceptos, procedimientos y relaciones subyacentes al propio objeto y a sus representaciones. En el caso particular de los intervalos no acotados de la recta real, en las reflexiones y recomendaciones que siguen hemos destacado explícitamente la complejidad del proceso infinito asociado a la no acotación del mismo, y proponemos tareas encaminadas a lograr ese aprendizaje.

En el caso de los intervalos no acotados de la recta real, las conversiones con mayor índice de congruencia han sido las conversiones entre los sistemas gráfico y numérico. Además, y como podemos ver en las Tablas 9 y 10, muchos de los valores mayores del IC involucran el registro gráfico. Así, en vista de los resultados del análisis de congruencia, afirmamos que la representación gráfica de estos intervalos puede ser una representación útil al iniciar el tratamiento del concepto. Además, esta representación muestra el intervalo como un subconjunto de la recta real, lo cual está muy ligado a su significado, aunque es necesario tener presentes las dificultades que pueden surgir al representar gráficamente números reales en la recta real (Scaglia y Coriat, 2003).

Por tanto, proponemos un tratamiento inicial del concepto en la docencia utilizando el registro gráfico, y la conversión del registro gráfico al numérico (y viceversa) como la primera coordinación que puede ser alcanzada. Esto podría efectuarse a través de tareas de conversión con intervalos tanto no acotados por la izquierda como por la derecha, y considerando diferentes valores posibles como extremos (números positivos, negativos, el cero, o un extremo con valor no concreto, "a"). Otras conversiones con un índice de congruencia alto en las Tablas 9 y 10 son algunas de las que involucran al registro gráfico con el registro verbal, y al registro gráfico con el algebraico (IC=46/80). En particular, aquellas en las cuales se mantiene el orden de aprehensión entre representaciones. De tal manera que si en las etapas iniciales se plantea una conversión del registro 
gráfico al verbal, o del gráfico al algebraico, puede ser más probable que los estudiantes de enseñanza secundaria recurran a la representación dentro del registro siguiendo el orden real (OR), coincidiendo con el orden de recorrido de la recta real en el registro gráfico, dado que así existe una mayor congruencia entre representaciones.

No obstante, es necesario consolidar posteriormente ese aprendizaje con el avance hacia coordinaciones con menor índice de congruencia, para disminuir gradualmente la posible elaboración por parte de los alumnos de conversiones aparentemente satisfactorias pero que sean puramente mecánicas, o basadas en la congruencia y la traducción de elementos constituyentes de la representación de un registro a otro. Esas estrategias de conversión no garantizan la existencia de una comprensión del concepto y pueden explicar los errores cometidos en situaciones con menor congruencia, como ya detectaron González-Calero et al. (2015) para el caso del error de inversión en la escritura de expresiones verbales y algebraicas en la resolución de problemas de enunciado verbal.

Para trabajar conversiones con menor congruencia, el docente ha de proponer conversiones en las que el orden de aprehensión no se mantenga. Por ejemplo, si se proporciona un intervalo no acotado por la derecha en el registro gráfico, y se pretende que los estudiantes lo conviertan al registro algebraico, puede añadirse la condición de que usen la desigualdad ">" en la expresión solicitada; o si es al registro verbal, la condición de que aparezca la palabra "mayor" o "mayores". O, si se parte de un registro sin univocidad representacional como el algebraico o el verbal, y se solicita su conversión a un registro como el gráfico o el numérico, proporcionar una representación en la que se utilice un orden lingüístico y no un orden real. Ejemplos de tarea podrían ser: "Representa gráficamente el intervalo $x>a$ " o "Representa gráficamente los números mayores que a".

Si además focalizamos nuestra atención en los intervalos no acotados, aparece la dificultad añadida del proceso infinito ligado a la no acotación del mismo. El conocimiento por parte de los alumnos de las conversiones entre representaciones, a priori, no nos asegura el aprendizaje de la caracterización de la no acotación de estos intervalos. Por tanto, también son necesarias tareas, en la secuencia de enseñanza, encaminadas a lograr ese aprendizaje. Un ejemplo de tarea puede ser el siguiente:

- Dado el intervalo $(3,+\infty)$, escribe si el número 10100 pertenece a este intervalo. Escribe un número mayor que 10100 que también pertenezca a este intervalo. Explica si pertenecen o no al intervalo todos los números 
mayores que 10100. Ahora, p es un número de este intervalo, determina otro número de este intervalo que sea mayor que $p$.

En las recomendaciones anteriores nos hemos centrado en actividades ligadas a la conversión entre representaciones como palanca importante de acceso al concepto y, también, en este caso concreto de los intervalos no acotados de la recta real, a la caracterización de la no acotación de los mismos. Esto no quiere decir que las actividades de aprendizaje deban reducirse al trabajo de conversiones entre representaciones, sino que también es necesario tratar otros aspectos, como la fenomenología del concepto o sus aplicaciones.

Para finalizar el artículo, destacamos las aportaciones del mismo y las líneas futuras que surgen, ligadas a este estudio teórico. A partir del reconocimiento de la importancia del dominio de las representaciones en el aprendizaje de un objeto matemático, y de la influencia del grado de congruencia en la dificultad de la conversión, hemos propuesto una metodología teórica para analizar el grado de congruencia entre representaciones de un objeto matemático. La metodología adapta los tres criterios señalados por Duval (1999), creándose además un criterio previo, la univocidad representacional, señalando una serie de pasos necesarios para desarrollar este tipo de análisis y definiendo un índice de congruencia que permita comparar la dificultad de las conversiones. Se ilustra la aplicación de la metodología en el caso de los intervalos de la recta real, un objeto matemático de difícil conceptualización en enseñanza secundaria, y cuyo aprendizaje ha sido poco investigado en didáctica de la matemática.

En el caso particular de los intervalos no acotados de la recta real, hemos utilizado los resultados obtenidos al aplicar esta metodología teórica de análisis para proponer una serie de reflexiones y recomendaciones didácticas a tener presentes en la generación de secuencias de enseñanza de este objeto matemático en la educación secundaria. Estos resultados y recomendaciones, desde el punto de vista teórico, abren otras investigaciones futuras sobre el concepto de intervalo, de tipo empírico, que lo deben complementar. Una de ellas sería analizar y detectar las estrategias de conversión entre representaciones de este objeto matemático utilizadas por los estudiantes, así como descubrir las posibles dificultades que pueden ponerse de manifiesto al hacer esas representaciones y conversiones. Esto nos permitiría, por una parte, comprobar si el éxito es mayor en las conversiones entre registros con mayor IC y, por otra, determinar qué estrategias de conversión, basadas o no en la congruencia, son empleadas. Otra estrategia consistiría en analizar los aprendizajes de los 
alumnos en una docencia sustentada en la aplicación práctica de secuencias de enseñanza basadas en los resultados expuestos en este trabajo y, con ello, determinar su efectividad y posible refinamiento. Finalmente, y dado que los intervalos son un prerrequisito muy importante para el desarrollo de la comprensión de tópicos y resultados propios del análisis matemático, sería interesante analizar cómo interpretan los estudiantes los intervalos que forman parte de los enunciados en los teoremas de análisis matemático.

\section{REFERENCIAS}

Adu-Gyamfi, K. y Bossé, M. J. (2014). Processes and reasoning in representations of linear functions. International Journal of Science and Mathematics Education, 12(1), 167-192.

Belmonte, J. L. y Sierra, M. (2011). Modelos intuitivos del infinito y patrones de evolución nivelar. Revista Latinoamericana de Investigación en Matemática Educativa, 14(2), 139-171.

Berciano, A., Ortega, T. y Puerta, M. (2015) Aprendizajes de las interpolaciones gráficas y algebraicas. Análisis comparativo. Enseñanza de las Ciencias, 33(3), 43-58.

Castro, E. y Castro, E. (1997). Representaciones y Modelización. En L. Rico (coord.), La Educación Matemática en la Enseñanza Secundaria (pp. 95-124). Barcelona, España: ICE-Horsori.

Clement, J. J. (1982). Algebra word problem solutions: Thought processes underlying a common misconception. Journal for Research in Mathematics Education, 13(1), 16-30.

Duval, R. (1999). Semiosis y pensamiento humano: registros semióticos y aprendizajes intelectuales. Cali, Colombia: Universidad del Valle, Instituto de Educación y Pedagogía, Grupo de Educación Matemática.

Duval, R. (2006). A cognitive analysis of problems of comprehension in a learning of Mathematics. Educational Studies in Mathematics, 61(1-2), 103-131.

Elia, I., Panaoura, A., Eracleous, A. y Gagatsis, A. (2007). Relations between secondary pupils' conceptions about functions and problem solving in different representations. International Journal of Science and Mathematics Education, 5(3), 533-556.

González-Calero, J. A., Arnau, D. y Laserna-Belenguer, B. (2015). Influence of additive and multiplicative structure and direction of comparison on the reversal error. Educational Studies in Mathematics, 89(1), 133-147.

Janvier, C. (ed.) (1987). Problems of representations in the teaching and learning of mathematics. Hillsdale, NJ: Lawrence Erlbaum Associated. 
Kaput, J. J. (1991). Notations and representations as mediators of constructive processes. En E. von Glasersfeld (ed.), Radical constructivism in Mathematics Education (pp. 53-74). Dordrecht, Holanda: Kluwer.

Laserna-Belenguer, B., Arnau, D. y González-Calero, J. A. (2014). La coincidencia del orden de las palabras como modelo explicativo al error de inversión. En J. L González, J. A. Fernández-Plaza, E. Castro-Rodríguez, M. T. Sánchez, C. Fernández, J. L. Lupiáñez y L. Puig (eds.), Investigaciones en Pensamiento Numérico y Algebraico e Historia de Ias Matemáticas y Educación Matemática - 2014 (pp. 101-108). Málaga: SEIEM.

Ministerio de Educación y Cultura (2007a). Real Decreto 1631/2006, de 29 de diciembre, por el que se establecen las enseñanzas mínimas correspondientes a la Educación Secundaria Obligatoria. Boletín Oficial del Estado 5, del 5 de enero de 2007 (pp. 677-773). Madrid, España: Gobierno de España.

Ministerio de Educación y Cultura (2007b). Real Decreto 1467/2007, de 2 de noviembre, por el que se establece la estructura del bachillerato y se fijan sus enseñanzas mínimas. Boletín Oficial del Estado 266, del 6 de noviembre de 2007 (pp. 4538145477). Madrid, España: Gobierno de España.

Ministerio de Educación, Cultura y Deporte (2015). Real Decreto 1105/2014, de 26 de diciembre, por el que se establece el currículo básico de la Educación Secundaria Obligatoria y del Bachillerato. Boletín Oficial del Estado 3, del 3 de enero de 2015 (pp. 169-546). Madrid, España: Gobierno de España.

Montoro, V., Cifuentes, M., Salva, N. y Bianchi, M. J. (2017). Estudiantes pensando en la recta numérica. Infancia y Aprendizaje: Journal for the Study of Education and Development, 40(2), 302-342.

NCTM (2000). Principles and standards for School Mathematics. Reston, Estados Unidos: NCTM.

OECD (2012). PISA 2012 Assessment and Analytical Framework. Mathematics, Reading, Science, Problem Solving and Financial Literacy. París, Francia: OECD.

Pecharromán, C. (2013). Naturaleza de los objetos matemáticos: representación y significado. Enseñanza de las Ciencias, 31(3), 121-134.

Rico, L. (1998). Errores y dificultades en el aprendizaje de las matemáticas. En J. Kilpatrick, P. Gómez y L. Rico (eds.), Educación Matemática. Errores y dificultades de los estudiantes. Resolución de problemas. Evaluación. Historia (pp. 69-108). Bogotá, Colombia: Una Empresa Docente.

Rico, L. (2009). Sobre las nociones de representación y comprensión en la investigación en educación matemática. PNA, 4(1), 1-14.

Scaglia, S. y Coriat, M. (2003). Dos conflictos al representar números reales en la recta. La Gaceta de la RSME, 6(1), 132-150. 
Socas, M. (2007). Dificultades y errores en el aprendizaje de las matemáticas. Análisis desde el Enfoque Lógico Semiótico. En M. Camacho, P. Flores y P. Bolea (eds.), Investigación en Educación Matemática XI (pp. 19-52). La Laguna, España: SEIEM.

\section{MATÍAS ARCE}

Dirección postal: Facultad de Educación de Segovia. Campus "María Zambrano". Universidad de Valladolid. Plaza de la Universidad, \#1, 40005 Segovia (España)

Teléfono: $\quad$ (+34)921112325 